\title{
Article \\ Cu-Ni-Based Alloys from Nanopowders as Potent Thermoelectric Materials for High-Power Output Applications
}

\author{
Mario Wolf ${ }^{1, *(\mathbb{D})}$, Jan Flormann ${ }^{1}$, Timon Steinhoff ${ }^{2}$, Gregory Gerstein ${ }^{2}$, Florian Nürnberger ${ }^{2}{ }^{(}$, \\ Hans Jürgen Maier ${ }^{2}(D)$ and Armin Feldhoff $1, *$ (D) \\ 1 Institute of Physical Chemistry and Electrochemistry, Leibniz University Hannover, Callinstraße 3A, \\ D-30167 Hannover, Germany; jan.flormann@pci.uni-hannover.de \\ 2 Institut für Werkstoffkunde (Materials Science), Leibniz University Hannover, An der Universität 2, \\ D-30823 Garbsen, Germany; steinhoff@iw.uni-hannover.de (T.S.); gerstein@iw.uni-hannover.de (G.G.); \\ nuernberger@iw.uni-hannover.de (F.N.); maier@iw.uni-hannover.de (H.J.M.) \\ * Correspondence: mario.wolf@pci.uni-hannover.de (M.W.); armin.feldhoff@pci.uni-hannover.de (A.F.)
}

check for updates

Citation: Wolf, M.; Flormann, J.;

Steinhoff, T.; Gerstein, G.;

Nürnberger, F.; Maier, H.J.; Feldhoff,

A. Cu-Ni-Based Alloys from

Nanopowders as Potent

Thermoelectric Materials for

High-Power Output Applications.

Alloys 2022, 1, 3-14. https:/ /

doi.org/10.3390/alloys1010002

Academic Editor: Nikki Stanford

Received: 1 December 2021

Accepted: 5 January 2022

Published: 11 January 2022

Publisher's Note: MDPI stays neutral with regard to jurisdictional claims in published maps and institutional affiliations.

Copyright: (C) 2022 by the authors. Licensee MDPI, Basel, Switzerland. This article is an open access article distributed under the terms and conditions of the Creative Commons Attribution (CC BY) license (https:// creativecommons.org/licenses/by/ $4.0 /)$.

\begin{abstract}
A new approach for the development of thermoelectric materials, which focuses on a high-power factor instead of a large figure of merit $z T$, has drawn attention in recent years. In this context, the thermoelectric properties of $\mathrm{Cu}-\mathrm{Ni}$-based alloys with a very high electrical conductivity, a moderate Seebeck coefficient, and therefore a high power factor are presented as promising low-cost alternative materials for applications aiming to have a high electrical power output. The Cu-Ni-based alloys are prepared via an arc melting process of metallic nanopowders. The heavy elements tin and tungsten are chosen for alloying to further improve the power factor while simultaneously reducing the high thermal conductivity of the resulting metal alloy, which also has a positive effect on the $z T$ value. Overall, the samples prepared with low amounts of Sn and W show an increase in the power factor and figure of merit $z \mathrm{~T}$ compared to the pure $\mathrm{Cu}-\mathrm{Ni}$ alloy. These results demonstrate the potential of these often overlooked metal alloys and the utilization of nanopowders for thermoelectric energy conversion.
\end{abstract}

Keywords: thermoelectric materials; $\mathrm{Cu}-\mathrm{Ni}$; alloying; thermal conductivity; power factor

\section{Introduction}

To achieve a sustainable electric power supply, the use of thermoelectric generators is a possible method to increase the energy efficiency in various applications by directly converting heat (waste) to electrical energy [1]. Thermoelectric materials have been intensely studied in recent decades, and promising candidates have been found for different applications [2]. The performance of a thermoelectric material is defined by the three central thermoelectric quantities, namely, the Seebeck coefficient $\alpha$, the electrical conductivity $\sigma$, and the open-circuited entropy conductivity $\Lambda$, which is related to the heat conductivity $\lambda=T \cdot \Lambda$ via the absolute temperature [3-6]. In the context of this work, thermal conductivity is used as a general term that can be expressed either by the heat conductivity $\lambda$ or by the more fundamental entropy conductivity $\Lambda$. From these thermoelectric quantities, the power factor $\sigma \alpha^{2}$, which can be used to calculate the maximum achievable power, and the figure of merit $f=z T$, which is used to calculate the maximum energy conversion efficiency, can be determined according to Equation (1) $[3,4,6]$. By using the entropy conductivity $\Lambda$ in Equation (1), the dimensionless figure of merit appears in a more concise form only containing the material parameters and it is not explicitly a function of the absolute temperature $T$, but implicitly, since all material parameters are temperature-dependent.

$$
f=\frac{\sigma \alpha^{2}}{\Lambda}=\frac{\sigma \alpha^{2}}{\lambda} \cdot T:=z T
$$


Thermoelectric materials are conventionally designed to maximize the figure of merit $z T$ and thus the energy conversion efficiency. For example, these can be achieved by nanostructuring or doping of promising materials [7-9]. For near room-temperature energy conversion, $\mathrm{Bi}_{2} \mathrm{Te}_{3}$ shows the most advanced properties $[10,11]$, but is characterized by major drawbacks: the toxicity of telluride compounds and the poor thermal stability. Consequently, various alternatives such as oxides [12-14] or intermetallic phases such as Zintl [15,16] or half-Heusler phases [17-19] are widely investigated. However, the focus on maximizing the figure of merit $z T$ is questionable [2,20], as the electrical power output of the material is determined by the power factor and not by the figure of merit $z T$ [21]. As a result, recent considerations have shown that to achieve a high electrical power output for high-temperature applications with a constant thermal gradient, a high power factor can be equally or more important than a high efficiency [2,20]. A promising approach towards thermoelectric material development is to focus on a material with a high electrical conductivity, such as one of the infrequently considered metal alloys, and then attempt to gradually improve the Seebeck coefficient and lower the thermal conductivity while maintaining high electrical conductivity to achieve a very high power factor. In Figure 1, Ioffe plots of type I (power factor vs. electrical conductivity) and type II (entropy conductivity vs. electrical conductivity) are displayed for comparison of common thermoelectric materials. Here, all relevant material parameters can be displayed in a concise form, giving a direct comparison and including information about the figure of merit due to the utilization of the entropy conductivity $\Lambda$ instead of the heat conductivity $\lambda$. In these plots, the thermoelectric properties are displayed as a function of their material parameters and rather than the absolute temperature $T$. This corresponds to the concise form of the figure of merit by utilization of the entropy conductivity. The aforementioned $\mathrm{Bi}_{2} \mathrm{Te}_{3}$ compounds show a very good power factor of 30 to $50 \mu \mathrm{W} \cdot \mathrm{cm}^{-1} \cdot \mathrm{K}^{-2}[10,22]$. Additionally, half-Heusler compounds such as $\mathrm{FeNb}_{0.88} \mathrm{Hf}_{0.12} \mathrm{Sb}$ [23] have been shown to reach a very high power factor of approximately 40 to $60 \mu \mathrm{W} \cdot \mathrm{cm}^{-1} \cdot \mathrm{K}^{-2}[19,23]$ and good $z T$ values, and they are among the most advanced materials in the field. However, within the Ioffe plots, another promising material from the group of metal alloys can be identified, when a high power output is the main goal: $\mathrm{Cu}-\mathrm{Ni}$ alloys. This elemental combination has been widely used in thermoelements as constantan ( $\mathrm{Cu}-\mathrm{Ni}-\mathrm{Mn})$ due to its properties and stability at elevated temperatures. It is characterized simultaneously by a very high power factor up to $100 \mu \mathrm{W} \cdot \mathrm{cm}^{-1} \cdot \mathrm{K}^{-2}$ (see Figure 1a) and a high thermal conductivity, showing potential for high-temperature applications where power output may be more relevant than conversion efficiency [24,25]. Cu-Ni alloys are especially promising candidates due to the resonant levels in their electronic structure, resulting in a remarkably high Seebeck coefficient [26]. Furthermore, Cu-Ni alloys show a strong deviation of the empirical Wiedemann-Franz relation, which is especially promising to be utilized for thermoelectric energy conversion [27]. Another major advantage of such alloys is that they consist of inexpensive and nontoxic elements, are characterized by a good thermal and mechanical stability, and they can be produced via large-scale metallurgic processes. The only drawback is the high thermal conductivity, which leads to a relatively low conversion efficiency (see Figure 1b). However, especially for high-temperature applications with low-cost heat sources, e.g., in the automotive sector or solar heat, and when a constant temperature gradient can be maintained, e.g., active cooling, the thermal conductivity plays a subordinate role and a high power factor is highly advantageous [2,20,28].

In this study, the above approach was employed, and the thermoelectric properties of $\mathrm{Cu}-\mathrm{Ni}$-based alloys were investigated. To date, only a few studies have investigated $\mathrm{Cu}-\mathrm{Ni}$ alloys for thermoelectric purposes. Some researchers tried to alter the material at the nanoscale by incorporating carbon nanotubes or $\mathrm{Al}_{2} \mathrm{O}_{3}$ nanoparticles into the alloy [24,29]. In both cases, the thermal conductivity could be significantly lowered, resulting in an increased $z T$ up to 0.35 at $800 \mathrm{~K}$ [29]. However, the incorporation also strongly reduced the electrical conductivity and therefore the power factor. Nanostructured and nanotwinned $\mathrm{Cu}-\mathrm{Ni}$ alloys were also investigated and were found to reach an exceptionally high power 
factor up to $100 \mu \mathrm{W} \cdot \mathrm{cm}^{-1} \cdot \mathrm{K}^{-2}$ at $900 \mathrm{~K}$ and a figure of merit of $0.18[25,30]$. Shimizu et al. presented a $\mathrm{Cu} / \mathrm{Cu}-\mathrm{Ni}$ thin film thermoelectric generator on a polyimide substrate; this film attained a remarkable power output of $21 \mu \mathrm{W}$ at a temperature difference of $70 \mathrm{~K}$ [31].
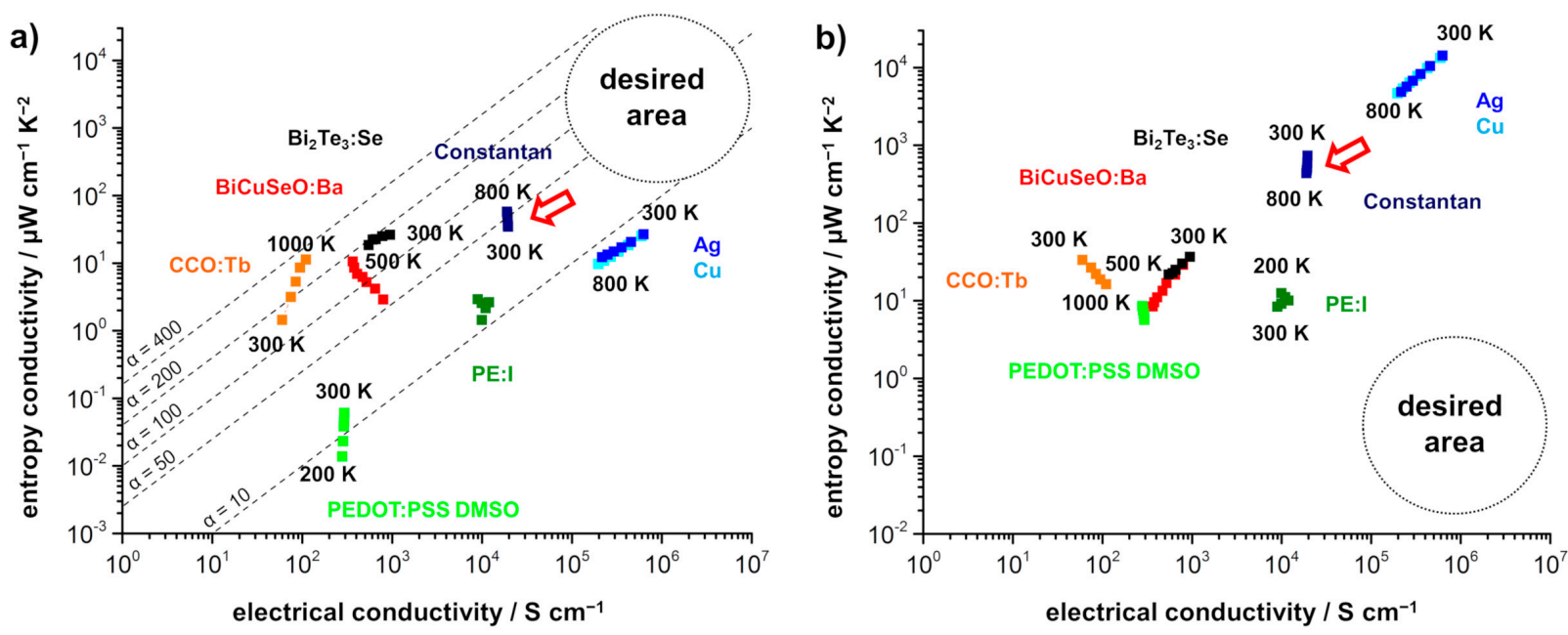

Figure 1. Ioffe plots for comparison of promising materials from various classes. (a) Type I Ioffe plot (power factor vs. electrical conductivity) and (b) type II Ioffe plot (entropy conductivity vs electrical conductivity). $\mathrm{Cu}-\mathrm{Ni}$ alloys are located at a very high power factor, making them promising materials for applications where high power output is desired. Adapted from [27], with permission from Springer, 2021.

The approach of this study was to alter the structure of the metal on the atomic level by alloying $\mathrm{Cu}-\mathrm{Ni}$ with heavier elements to beneficially influence the power factor and simultaneously reduce the thermal conductivity via long-range phonon scattering. Specifically, $\mathrm{Sn}$ and $\mathrm{W}$ were chosen as metals; they are both heavier than $\mathrm{Ni}$ and $\mathrm{Cu}$ but have quite different properties. While $\mathrm{Sn}$ has a low melting point of $505 \mathrm{~K}$ [32] and mixes well with $\mathrm{Cu}$ (bronzen), W has a much higher melting point of $3695 \mathrm{~K}$ [32] and is not incorporated in the matrix [33]. The degree to which these different properties influence the resulting microstructure and the thermoelectric properties was studied. All alloys have been prepared from metal nanopowders via arc melting, in contrast to our previous report [34] that presented the idea of $\mathrm{Cu}-\mathrm{Ni}$ alloys from larger flakes resulting in more inhomogeneous distribution and inferior thermoelectric properties. By this, the influence of the utilization of nanopowders as well as the results of alloying with $\mathrm{Sn}$ and $\mathrm{W}$ are investigated.

\section{Materials and Methods}

If not mentioned otherwise, all metals were obtained from commercial vendors at 99.9\% purity or higher and are used without further purification. $\mathrm{Cu}, \mathrm{Sn}$, and $\mathrm{W}$ nanopowders (average diameter less than $100 \mathrm{~nm}$ ) were obtained from IoLiTec Nanomaterials (Heilbronn, Germany), and Ni (average diameter less than $100 \mathrm{~nm}$ ) was obtained from Sigma-Aldrich (Taufkirchen, Germany). These powders were stored and processed under an inert gas (argon) atmosphere in a glove box. Metallic precursor mixtures were prepared by mixing, agitating, and shaking under argon with the stoichiometric ratios listed in Table 1. The resulting powders were pressed into discs using a hydraulic press; they were subsequently arc-melted in a copper crucible to form ingots by means of an EWM TETRIX 521 CLASSIC activArc TIG welding machine (EWM, Mündersbach, Germany) that was operated for $30 \mathrm{~s}$ with a current of $150 \mathrm{~A}$ and an argon flow of $5 \mathrm{~L} \cdot \mathrm{min}^{-1}$. 
Table 1. Designations and atomic compositions of the prepared samples in atomic percent $x$ of the respective metallic nanopowders. For comparison, a pure $\mathrm{Cu}-\mathrm{Ni}$ alloy with a 50:50 ratio is prepared (Cu50Ni50). For the Sn-alloys, samples with 1 at.\% (Sn1), 2 at.\% (Sn2), and 5 at.\% (Sn5) and for the W-alloys, samples with 1 at.\% (W1), 5 at.\% (W5), and 10 at.\% (W10) with Sn or W were prepared, respectively. All samples have been prepared from nanopowders with an average diameter less than $100 \mathrm{~nm}$.

\begin{tabular}{ccccc}
\hline Sample & $x \mathbf{( C u})$ & $x \mathbf{( N i )}$ & $x(\mathbf{S n})$ & $x(\mathbf{W})$ \\
\hline Cu50Ni50 & 50 & 50 & - & - \\
Sn1 & 49.5 & 49.5 & 1 & - \\
Sn2 & 49 & 49 & 2 & - \\
Sn5 & 47.5 & 47.5 & 5 & 1 \\
W1 & 49.5 & 49.5 & - & 5 \\
W5 & 47.5 & 47.5 & - & 10 \\
W10 & 45 & 45 & - & \\
\hline
\end{tabular}

For the measurement of the Seebeck coefficient and the electrical conductivity, rodshaped samples ( $3 \mathrm{~mm}$ diameter and $10 \mathrm{~mm}$ length) were cut with a Sodick AD325L wire electrical discharge machine (EDM, Sodick Deutschland $\mathrm{GmbH}$, Düsseldorf-Lichtenbroich, Germany) unit. The microstructure and composition were characterized by X-ray diffraction (XRD, Bruker D8 Advance with $\mathrm{Cu}-\mathrm{K} \alpha$ radiation, Bruker AXS GmbH, Karlsruhe, Germany) of the polished alloys and by field-emission scanning electron microscope (FE-SEM, ZEISS Supra 55 VP, Carl Zeiss Microscopy Deutschland GmbH, Oberkochen, Germany) of polished cross-sections. The electron microscope was additionally equipped with an energy-dispersive X-ray spectrometer (EDXS System Quantax from Bruker, Bruker AXS GmbH, Karlsruhe, Germany). The electrical conductivity $\sigma$ was determined with a custom-made measurement cell with an ELITE thermal system and KEITHLEY 2100 digital multimeters (Keithly Instruments, Solon, OH, USA). The Seebeck coefficient $\alpha$ was measured using a ProbotStat A apparatus from NORECS AS (Haslevollen, Norway). The density was then determined at room temperature by using the Archimedes method with isopropanol as solvent. The thermal diffusivity was measured using a light flash analyzer (Netzsch LFA 467 HT, Netzsch-Gerätebau GmbH, Selb, Germany) from room temperature to $600 \mathrm{~K}$. The heat capacity was calculated using the Dulong-Petit law.

\section{Results and Discussion}

XRD patterns of the Cu-Ni-based alloys are shown in Figure 2. For the Cu50Ni50 sample, good agreement with the literature data for $\mathrm{Cu}-\mathrm{Ni}$ alloys is apparent. Small additional reflections marked with an asterisk correspond to minor $\mathrm{NiO}$ impurities, most likely due to the utilization of nanopowders, which are especially susceptible to oxidation due to their high surface area. This impurities can be identified for all Sn-alloyed samples, but cannot be identified for the 5 at.\% and the 10 at.\% W samples. For the Sn-alloyed samples, the observed 111 and $200 \mathrm{Cu}-\mathrm{Ni}$ reflections are shifted towards lower angles as indicated by vertical dashed lines. This can be explained by an incorporation of the $\mathrm{Sn}$ atoms into the $\mathrm{Cu}-\mathrm{Ni}$ matrix, resulting in a larger lattice parameter. With increasing amount of Sn, this shift to lower angles becomes more apparent (Figure 2a). Additionally, no pure $\mathrm{Sn}$ inclusion can be identified. In the $\mathrm{W}$-alloyed samples, the $\mathrm{Cu}-\mathrm{Ni}$ reflections are not shifted, as again indicated by vertical dashed lines, and additional reflections can be observed for the 10 at.\% $\mathrm{W}$ sample, which match the reflections expected for pure $\mathrm{W}$ from the literature (Figure $2 \mathrm{~b}$ ). This indicates that $\mathrm{W}$ is not incorporated into the matrix, but forms a separate phase, which is in good agreement with the much higher melting point and the previous results of the flake-based samples [34]. As a result, Sn seems to be at least partly incorporated into the matrix, while tungsten primarily forms inclusion. For the alloys, changed ratios of the intensities of the 111 and 200 reflections can be detected, e.g., shown in the Sn2 sample, which is based on a preferred orientation due to directed solidification in the arc-melting process. This has been reported before for arc-melted $\mathrm{Cu}-\mathrm{Ni}$ 
alloys $[25,30]$ but with minor influence on the thermoelectric properties due to the isotropic fcc crystal structure [30].

a)

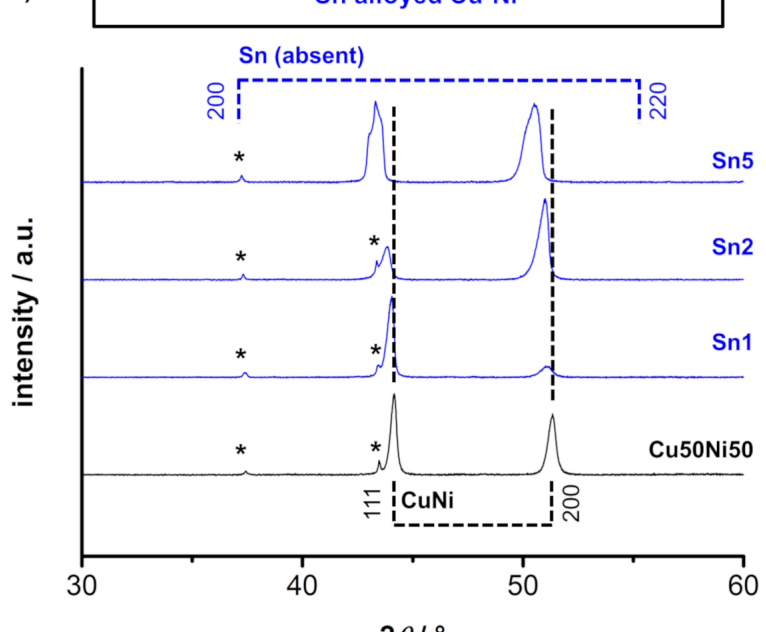

b)

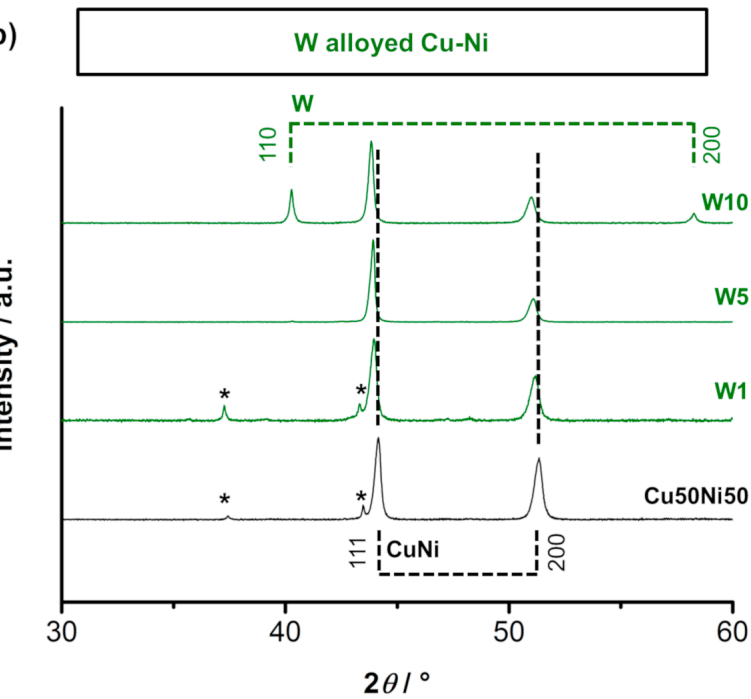

Figure 2. Normalized XRD patterns of the prepared samples from nanopowders. (a) Sn-alloyed and (b) W-alloyed Cu-Ni samples. Positions of the expected reflections for Cu-Ni (PDF 01-077-7711, black), Sn (PDF 01-077-3457, blue), and W (PDF 01-080-3012, green) are included and marked via vertical dashed lines, which are grouped by horizontal lines. Reflections marked with an asterisk correspond to NiO impurities (PDF 01-089-5881). The black dashed lines show the 111 and $200 \mathrm{Cu}-\mathrm{Ni}$ reflections of the undoped $\mathrm{Cu}-\mathrm{Ni}$ to highlight the occurring shift in the Sn alloyed samples compared to the $\mathrm{W}$ alloyed samples. For the Sn samples, this shift indicates the incorporation of $\mathrm{Sn}$ in the matrix. In the $\mathrm{W}$ samples, $\mathrm{W}$ can be identified as an additional phase that is not incorporated in the matrix.

Backscattered electron (BSE) micrographs and EDXS elemental mappings for $\mathrm{Cu}, \mathrm{Ni}$, $\mathrm{Sn}$, and $\mathrm{W}$ of the Cu50Ni50 sample and the Sn-alloyed samples are shown in Figure 3. The BSE micrograph of the Cu50Ni50 sample shows a mostly homogeneous distribution and some darker spots indicating lighter elements such as oxygen. Alongside the small impurities found in the XRD pattern, this could be caused by $\mathrm{NiO}$ inclusions. Within the EDXS mapping, the mostly purple coloration is assumed to indicate a relatively homogenous distribution of $\mathrm{Ni}$ (blue) and $\mathrm{Cu}$ (red). This shows a significantly improved elemental distribution due to the utilization of nanopowders, compared to the large flake-based samples [34]. However, some areas with increased $\mathrm{Cu}$ or $\mathrm{Sn}$ concentrations are still recognizable. On closer inspection, it is noticeable that $\mathrm{Sn}$ mixes preferentially with $\mathrm{Cu}$ (reddish-turquoise spots), while there is less mixing of $\mathrm{Ni}$ and $\mathrm{Sn}$ (blueish-magenta spots). This indicates that on a macroscopic scale, $\mathrm{Sn}$ is at least partly incorporated into the $\mathrm{Cu}-\mathrm{Ni}$ matrix. Additionally, small lighter spots are recognizable within the BSE micrographs, which correspond to Sn-rich areas according to the EDXS elemental analysis. Although the XRD patterns show that $\mathrm{Sn}$ is incorporated into the structure, some additional small Sn-rich spots are also formed. Hence, Sn seems to be partly incorporated and partly separated in another phase. This is most likely the result of two factors: First, the mixture of the nanopowders was prepared by mixing, agitating, and shaking under inert gas. This may not result in a homogeneous mixture on the nanoscale and may be improved by dispersing the powders in an organic solvent with a subsequent drying step or via a bottom-up synthesis. Second, the relatively short time of the arc-melting compared to annealing the alloys for several hours does not allow the molten particles much time for better distribution and incorporation. The Sn-alloyed samples also exhibit dark blue, Ni-rich spots. Here, a beginning dendritic growth of this inclusion becomes apparent, which has been reported for $\mathrm{NiO}$ before [35-37]. It is assumed, that the formation of $\mathrm{NiO}$ is also a result of the utilization of nanopowders, which are susceptible to oxidation due to their high surface area. 

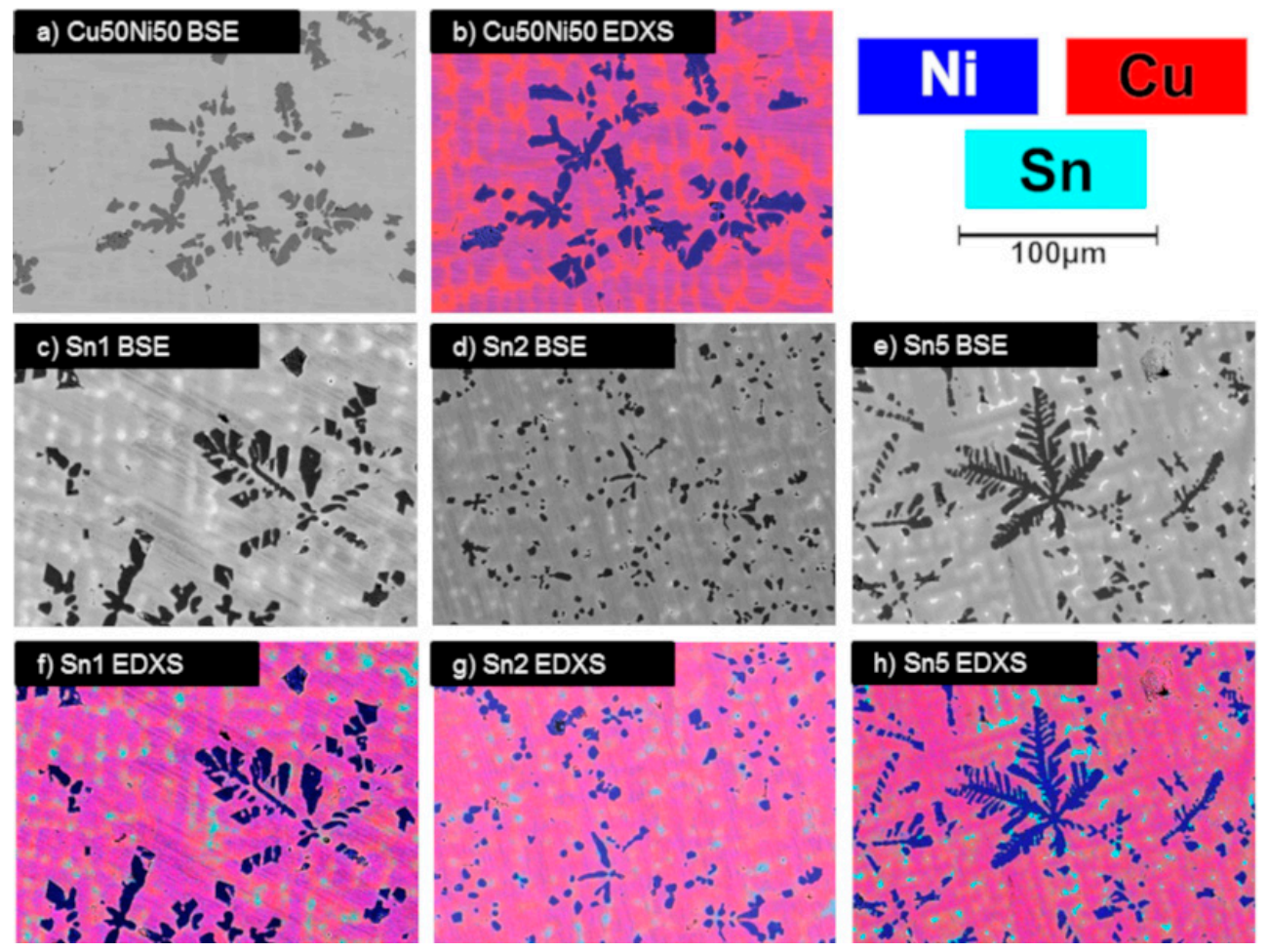

Figure 3. SEM BSE micrographs and EDXS elemental mappings of prepared Sn-alloyed samples from nanopowders. (a) BSE micrograph and (b) EDXS elemental mapping of the Cu50Ni50 sample, (c-e) BSE micrographs and (f-h) EDXS elemental mappings of the Sn-alloyed samples. $\mathrm{Cu}(\mathrm{red})$ and $\mathrm{Ni}$ (blue) form the matrix, in which Sn (cyan) is incorporated.

The measured temperature-dependent thermoelectric properties of the Sn-alloyed samples are shown in Figure 4. Here, an additional comparison to the constantan alloy $\left(\mathrm{Cu}_{56} \mathrm{Ni}_{42} \mathrm{Mn}_{2}\right)$ reported from Mao et al. [25] was chosen (dashed lines), as they also used a top-down approach via ball-milling with subsequent arc-melting, resulting in the best comparability. In the following, this literature sample for comparison is referred to as constantan. The Cu50Ni50 sample shows a similar electrical conductivity compared to constantan around $20,000 \mathrm{~S} \cdot \mathrm{cm}^{-1}$ with weak temperature dependence. After alloying with 1 at.\% Sn (Sn1), an increased electrical conductivity between 23,500 and 21,500 S. $\mathrm{cm}^{-1}$ could be reached (Figure 4a). For 2 at.\% Sn (Sn2), the electrical conductivity is again slightly increased, up to $24,500 \mathrm{~S} \cdot \mathrm{cm}^{-1}$ at $300 \mathrm{~K}$. Afterwards, it decreases with increasing amount of $\mathrm{Sn}$, equaling the Cu50Ni50 sample with 5 at.\% Sn, as further Sn addition forms more and more inclusions. All prepared samples show a more metallic behavior, with a decreasing electrical conductivity with increasing temperature. The higher electrical conductivity of the $\mathrm{Sn} 1$ and $\mathrm{Sn} 2$ samples is assumed to be a result of the incorporation of the Sn into the matrix, which directly influences the charge carrier concentration. The Seebeck coefficient of the Sn1 sample is slightly higher compared to Cu50Ni50 and then also shows a slightly decreasing behavior with increasing amounts of Sn (Figure $4 \mathrm{~b}$ ). The resulting power factor (Figure $4 \mathrm{c}$ ) of $38 \mu \mathrm{W} \cdot \mathrm{cm}^{-1} \cdot \mathrm{K}^{-2}$ at $573 \mathrm{~K}$ for the Sn1 sample is therefore about $12 \%$ higher compared to the $\mathrm{Cu} 50 \mathrm{Ni50}$, surpassing the aforementioned half-Heusler compounds, which usually show a power factor around 20 to $30 \mu \mathrm{W} \cdot \mathrm{cm}^{-1} \cdot \mathrm{K}^{-2}$ in this temperature region [19]. Compared to constantan, however, the resulting power factor is lower due to the diminished Seebeck coefficient. The density of the metal alloys (Figure $4 \mathrm{~d}$ ) increases as a result of the $\mathrm{Sn}$ inclusion for the $\mathrm{Sn} 1$ sample and also shows a decreasing behavior with increasing amounts of Sn. The values for the heat conductivity of the Sn-alloyed samples are shown in Figure 4e. With 1 at.\% Sn, the heat conductivity is slightly higher compared to the Cu50Ni50 sample, due to the much higher electrical conductivity. With increasing amounts of $\mathrm{Sn}$, the heat conductivity decreases as expected, as a result of the decreasing 
electrical conductivity and long-range phonon scattering at the inclusions. Compared to constantan, the samples are characterized by a significantly lower thermal conductivity, probably also due to the utilization of nanopowders. Finally, the resulting figure of merit is displayed in Figure $4 \mathrm{f}$. As a result of the increased electrical conductivity, the $z T$ of the Sn1 sample is slightly higher compared to the Cu50Ni50, but slightly lower compared to constantan, reaching a $z T$ value up to 0.09 at $573 \mathrm{~K}$. The $z T$ value of the $\mathrm{Cu} 50 \mathrm{Ni50}$ is therefore also slightly lower compared to constantan, as it is characterized by a lower Seebeck coefficient, which is not fully compensated by the lower thermal conductivity.
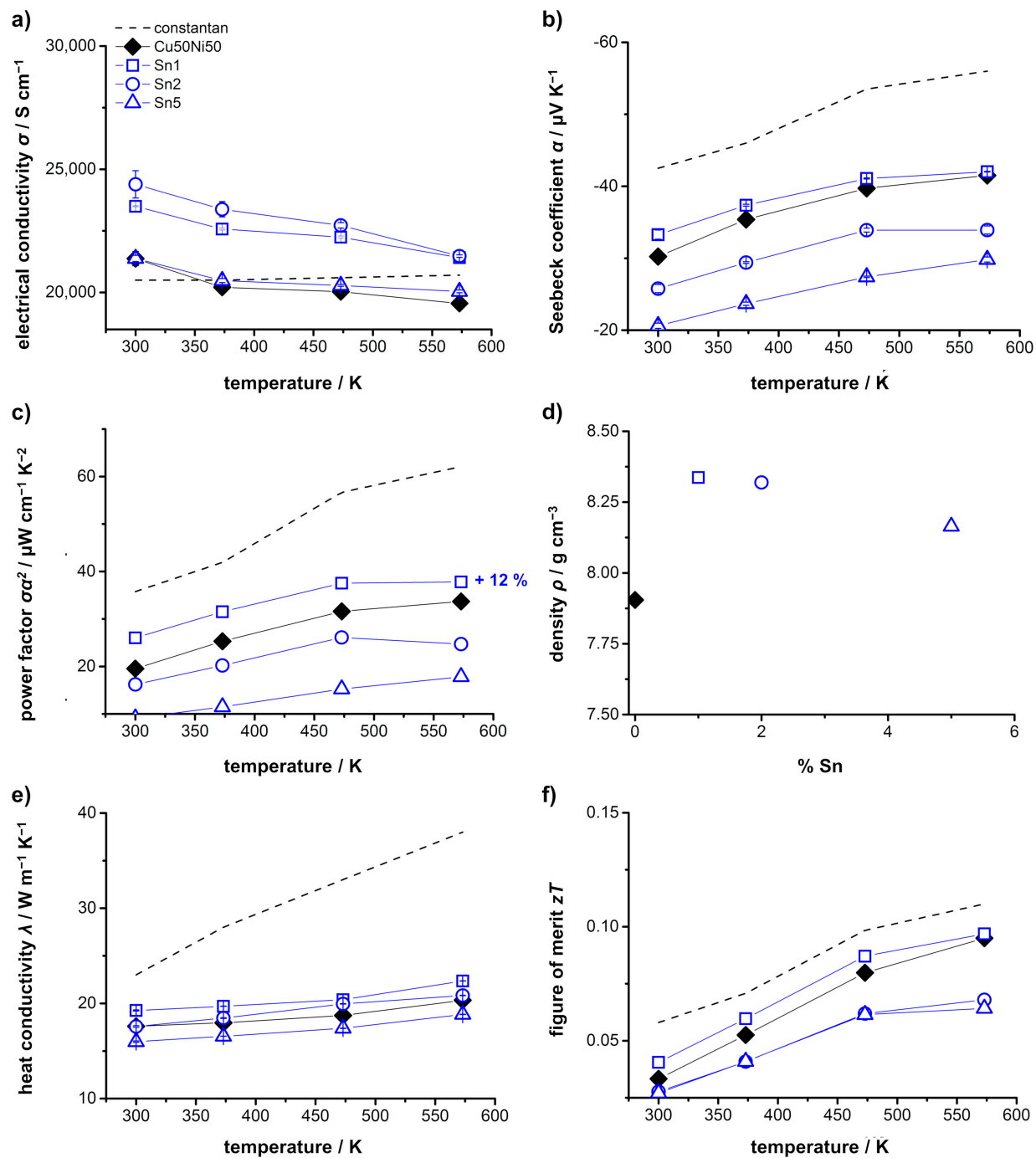

Figure 4. Measured temperature-dependent thermoelectric properties of the Sn-alloyed samples from nanopowders. (a) Isothermal electrical conductivity $\sigma$; (b) Seebeck coefficient $\alpha$, note the reversed vertical axis; (c) resulting power factor $\sigma \alpha^{2} ;(\mathbf{d})$ density $\rho$; (e) heat conductivity $\lambda$; (f) resulting figure of merit $z T$. Error bars are included for the measurement of the electrical conductivity, Seebeck coefficient and heat conductivity. In particular, the Sn1 sample shows an increased electrical conductivity. The power factor is slightly higher compared to the Cu50Ni50 sample, but lower compared to the constantan $\left(\mathrm{Cu}_{56} \mathrm{Ni}_{42} \mathrm{Mn}_{2}\right)$ (data from Mao et al. [25]). The heat conductivity slightly decreases with increasing amount of Sn. The resulting figure of merit $z T$ of the Sn1 sample is slightly higher compared to the Cu50Ni50 sample, but slightly lower compared to constantan.

SEM microstructural analyses of the $\mathrm{W}$-alloyed $\mathrm{Cu}-\mathrm{Ni}$ samples are shown in Figure 5. The distribution of $\mathrm{Cu}$ and $\mathrm{Ni}$ is analogous to the $\mathrm{Sn}$-alloyed samples and again $\mathrm{NiO}$ inclusions can be seen in all samples. In accordance to the discussion of the XRD patterns, 
the $\mathrm{W}$ seems to be not incorporated into the matrix and can be clearly identified as lighter spots in the BSE micrographs. With increasing amount of W, more of these spots become apparent. This is confirmed by the EDXS elemental mappings of these samples, where sharply defined green spots are recognizable; they also show that $\mathrm{W}$ forms its own pure phase and is not part of the matrix.
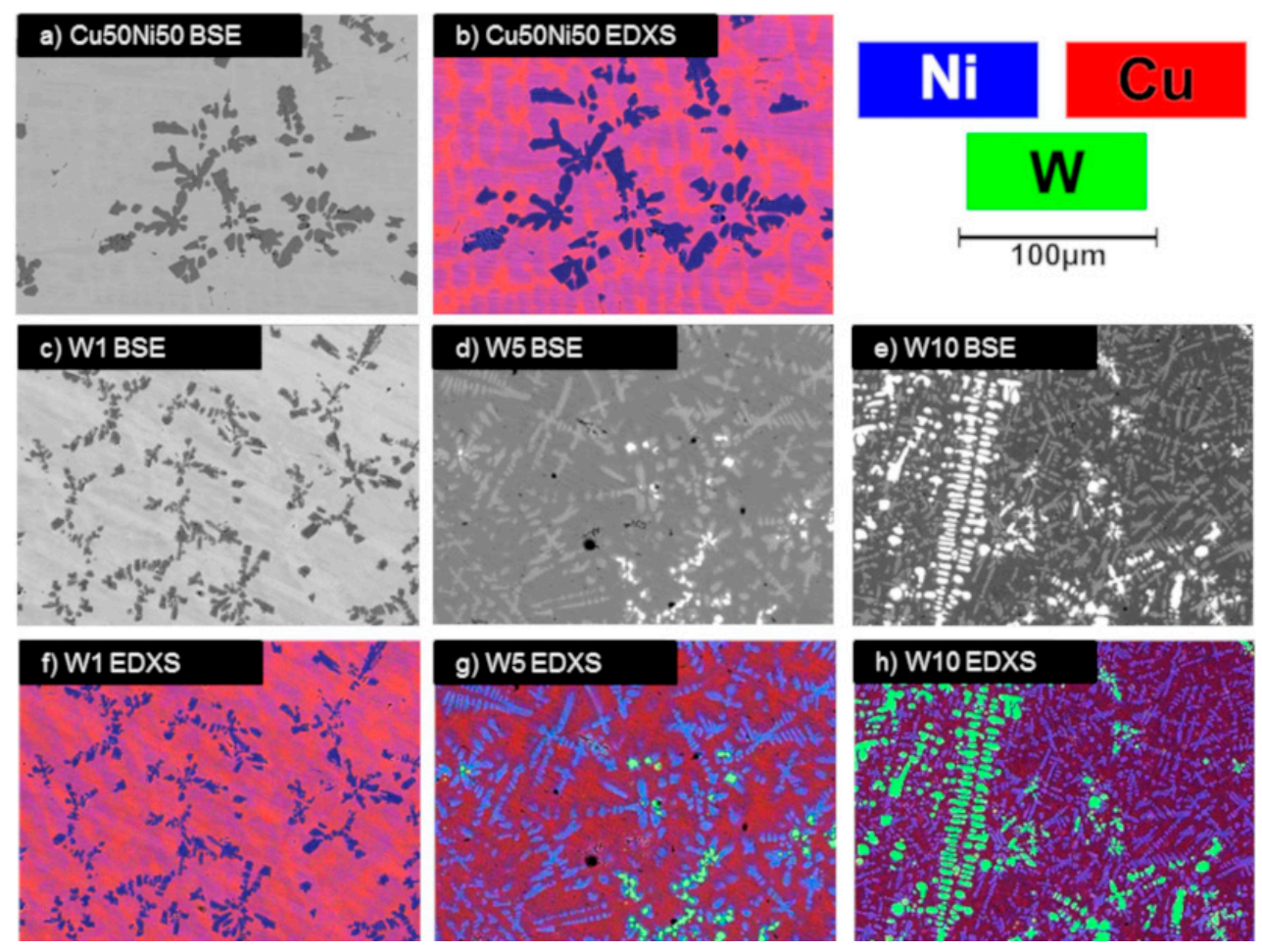

Figure 5. SEM BSE micrographs and EDXS elemental mappings of prepared W-alloyed samples from nanopowders. (a) BSE micrograph and (b) EDXS elemental mapping of the Cu50Ni50 sample, (c-e) BSE micrographs and (f-h) EDXS elemental mappings of the $\mathrm{W}$ alloyed samples. $\mathrm{Cu}(\mathrm{red})$ and $\mathrm{Ni}$ (blue) form the matrix, in which $\mathrm{W}$ (green) is not incorporated.

The measured temperature-dependent thermoelectric properties of the $\mathrm{W}$-alloyed samples are shown in Figure 6. A comparison to constantan is again shown via dashed lines. The electrical conductivity slightly decreases as a result of the $\mathrm{W}$ inclusions and with increasing amount of W (Figure 6a). The Seebeck coefficient of the W1 sample is slightly higher compared to the Cu50Ni50 sample and similar to constantan (Figure 6b). However, with increasing amount of $\mathrm{W}$, the Seebeck coefficient shows the same decrease as in the Sn samples. As a result, the power factor (Figure $6 \mathrm{c}$ ) of the W1 sample is also higher compared to the Cu50Ni50 sample (about 38\%), due to the higher Seebeck coefficient, but decreases with increasing amount of W. This behavior is analogous to the Sn-alloyed samples; however, here the increased power factor is a result of a higher Seebeck coefficient, while in the Sn-alloyed samples it is based on a higher electrical conductivity. Compared to constantan, the power factor is slightly lower. The values of the sample density also show a similar behavior as the Sn-alloyed samples; first it shows an increase up to 5 at. $\%$ and then decreases to the value of the Cu50Ni50 sample (Figure $6 \mathrm{~d}$ ). The measured heat conductivity again decreases with increasing amounts of $\mathrm{W}$, as expected, due to the decreasing electrical conductivity and long-range phonon scattering due to the inclusions (Figure 6e). Here, the alloying with 1 at.\% W results in a figure of merit of approximately 0.12 at $573 \mathrm{~K}$, corresponding to an increase of about $26 \%$ compared to the Cu50Ni50 sample. (Figure $6 \mathrm{f}$ ). 

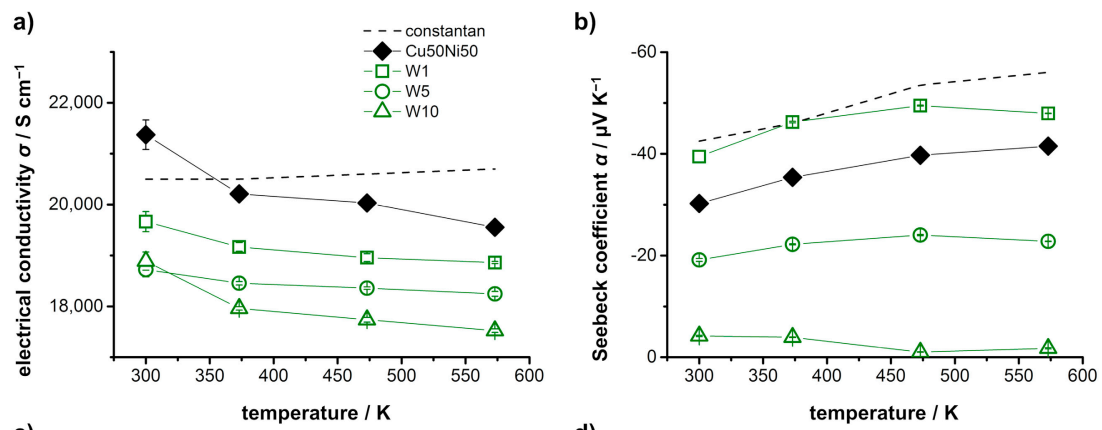

c)

d)
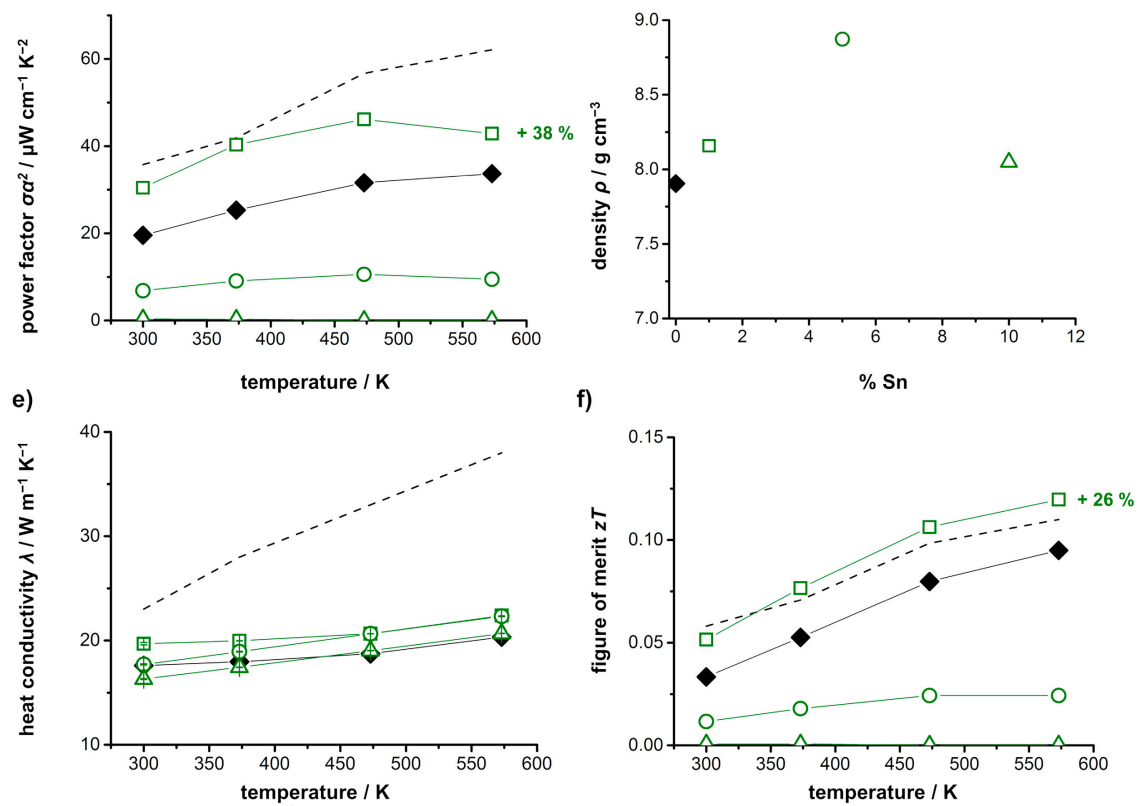

Figure 6. Measured temperature-dependent thermoelectric properties of the $\mathrm{W}$-alloyed samples from nanopowders. (a) Isothermal electrical conductivity $\sigma$; (b) Seebeck coefficient $\alpha$, note the reversed vertical axis; (c) resulting power factor $\sigma \alpha^{2} ;$ (d) density $\rho$; (e) heat conductivity $\lambda$; (f) resulting figure of merit $z T$. Error bars are included for the measurements of the electrical conductivity, Seebeck coefficient and heat conductivity. The W1 sample shows the highest power factor, which is slightly higher compared to the Cu50Ni50 sample, but slightly lower compared to constantan (data from [25]). The heat conductivity of all samples is again reduced, resulting in a higher figure of merit $z T$ of the W1 sample compared to the literature reference and the Cu50Ni50 sample.

Figure 7 summarizes the measured thermoelectric properties of the Sn-alloyed and Walloyed Cu-Ni samples. In the type I Ioffe plot (Figure 7a), the power factor of the samples is shown as a function of the electrical conductivity. Both, the Sn1 and W1 sample exhibit a higher power factor compared to the Cu50Ni50 sample, but slightly lower values compared to the reported ones for constantan. The Sn-alloyed samples show a strong increase in the electrical conductivity, most likely due to the incorporation of the Sn into the matrix and the consequent influence on the charge carrier concentration. The $\mathrm{W}$-alloyed samples are characterized by a higher Seebeck coefficient, but a decreased electrical conductivity as it is not incorporated and primarily forms inclusions. In the type II Ioffe plot (Figure $7 \mathrm{~b}$ ), the entropy conductivity is displayed as a function of the electrical conductivity. All prepared samples have a lower thermal conductivity compared to constantan, which is based on the alloying with $\mathrm{Sn}$ and $\mathrm{W}$ resulting in enhanced phonon scattering, especially at the inclusions, and on the utilization of nanopowders. With increasing amounts of Sn and $\mathrm{W}$, the thermal conductivity further decreases slightly. The resulting figure of merit of all prepared samples is shown in Figure 7c. For W-alloying, the respective sample with 1 at.\% 
is characterized by an increased $z T$ value of 0.12 , while the Sn-alloying resulted in similar $z T$ value of 0.09 .
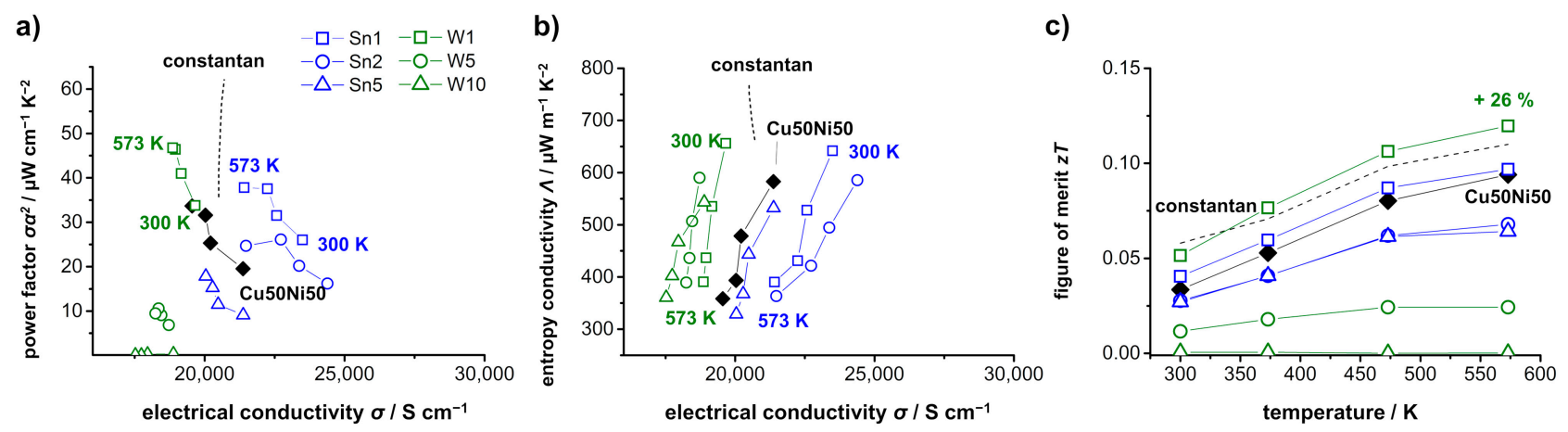

Figure 7. Summarized thermoelectric properties of the investigated samples. (a) Power factor as a function of the electrical conductivity (type I Ioffe plot). (b) Entropy conductivity as a function of the electrical conductivity (type II Ioffe plot). (c) Resulting temperature-dependent figure of merit $z T$ for Sn and W alloying. The power factor could be increased by low amounts of Sn and W compared to the pure $\mathrm{Cu}-\mathrm{Ni}$ alloy, but is lower compared to constantan (data from [25]). The energy conversion efficiency could be improved in comparison to both, the pure $\mathrm{Cu}-\mathrm{Ni}$ alloy and constantan with low amounts of Sn and W.

Overall, $\mathrm{Cu}-\mathrm{Ni}$ alloys containing $\mathrm{Sn}$ and $\mathrm{W}$ could be prepared. Due to the utilization of nanopowders, the distribution within the alloys could be significantly improved compared to large flake-based samples [34]. However, the distribution and homogeneity of the Sn and $\mathrm{W}$ can still be further enhanced, as both can still be identified as inclusions, although especially Sn should be incorporated in the Cu-Ni matrix. To improve this, other or multiple melting steps or additional annealing steps could be applied. Additionally, formation of $\mathrm{NiO}$ impurities could be found (see Figures 2, 3 and 5), which also might influence the resulting thermoelectric properties. Generally, these impurities should lead to a reduced electrical conductivity, due to the insulating character of $\mathrm{NiO}$, but simultaneously also to a lower thermal conductivity. Here, the latter has been found for all investigated alloys. For the electrical conductivity, however, the expected decrease can be identified for the W-alloyed sample, while alloying with small amount of Sn shows an increase in electrical conductivity, due to the incorporation of Sn into the matrix. If these impurities can be avoided, the thermoelectric properties and especially the power factor may be improved even further. In general, alloying with only small amounts, namely, 1 at.\% Sn or W, shows a beneficial behavior. For these samples, a higher power factor of 38 and $47 \mu \mathrm{W} \cdot \mathrm{cm}^{-1} \cdot \mathrm{K}^{-2}$ and $z T$ values of 0.09 and 0.12 were determined, respectively, corresponding to an increase of approximately 12 and $38 \%$ in the power factor compared to the Cu50Ni50 sample and $26 \%$ increase in the figure of merit for the $\mathrm{W}$-alloyed sample. Here, alloying with Sn resulted mainly in an increase in electrical conductivity, while alloying with $\mathrm{W}$ resulted in an increase of the Seebeck coefficient. This proves the potential of utilizing nanopowders, as a preparation from large metal flakes did not result in improved properties [34]. Compared to other works, Kang et al. [30] also prepared constantan with a nominal composition of $\mathrm{Cu}_{56} \mathrm{Ni}_{42} \mathrm{Mn}_{2}$ doped with 0.25 at.\% $\mathrm{Zr}$ via ball milling and induction melting, resulting in similar values to constantan for the undoped samples and increased values for $\mathrm{Zr}$-doped samples with a power factor of $80 \mu \mathrm{W} \cdot \mathrm{cm}^{-1} \cdot \mathrm{K}^{-2}$ and a peak $z T$ of 0.22 at $800 \mathrm{~K}$ after aging. Yuan et al. [29] prepared a $\mathrm{Cu}_{55} \mathrm{Ni}_{45} \mathrm{Se} /$ carbon nanotube hybrid material via ball milling and annealing, resulting in a $z T$ value of 0.35 at $873 \mathrm{~K}$, strongly increased by the inclusion of the carbon nanotubes, which leads to a drastically lowered thermal conductivity. Here, the results of this work are slightly lower, but show the beneficial characteristics by alloying with other elements and the utilization of nanopowders, which could be consequently combined with other concepts. These results underline the potential of these mostly overlooked materials; by alloying with other metals, the thermoelectric properties can be 
further enhanced and may even reach a moderate figure of merit $z T$, under maintaining a high power factor within an inexpensive, nontoxic, and easily obtainable material.

\section{Conclusions}

$\mathrm{Cu}-\mathrm{Ni}$-based metals alloyed with tin and tungsten were successfully produced via the arc melting of metal nanopowders. XRD and SEM analyses showed that tin is partly incorporated into the matrix, while tungsten primarily forms a separate phase. The thermal conductivity is slightly reduced by both alloying elements, whereas the power factor could be enhanced by small amounts of tin and tungsten compared to the pure $\mathrm{Cu}-\mathrm{Ni}$ alloy. The largest improvement was observed for samples with 1 at.\% tin or tungsten, resulting in a peak power factor of $38 \mu \mathrm{W} \cdot \mathrm{cm}^{-1} \cdot \mathrm{K}^{-2}$ and $47 \mu \mathrm{W} \cdot \mathrm{cm}^{-1} \cdot \mathrm{K}^{-2}$ for 1 at. $\%$ Sn and 1 at.\% W at $573 \mathrm{~K}$. In particular, the utilization of nanopowders resulted in better distribution and increased thermoelectric properties. Remarkably, Sn alloying mainly led to an increase in electrical conductivity, while W alloying increased the Seebeck coefficient. Within all samples, $\mathrm{NiO}$ impurities could be found. If they are avoided, these values are expected to be improved even further. These results showed that $\mathrm{Cu}-\mathrm{Ni}$ alloys are an interesting starting point for the development of a new generation of inexpensive, nontoxic, easily obtainable and processable thermoelectric materials with a very high power factor to be utilized for high power output.

Author Contributions: F.N., H.J.M. and A.F. worked on the conceptualization; G.G. and M.W. developed the methods and carried out the planning of the samples. M.W., J.F. and T.S. prepared and measured the samples. M.W. and J.F. prepared the original draft. All authors critically revised the manuscript. All authors have read and agreed to the published version of the manuscript.

Funding: This research received no external funding. The publication of this article was funded by the Open Access fund of the Leibniz University Hannover.

Institutional Review Board Statement: Not applicable.

Informed Consent Statement: Not applicable.

Data Availability Statement: There is no public available support of the data.

Conflicts of Interest: The authors declare no conflict of interest.

\section{References}

1. Kishore, R.A.; Marin, A.; Wu, C.; Kumar, A.; Priya, S. Energy Harvesting-Materials, Physics, and System Design with Practical Examples; DEStech Publications: Lancaster, PA, USA, 2019.

2. Wolf, M.; Hinterding, R.; Feldhoff, A. High Power Factor vs. High zT-A Review of Thermoelectric Materials for HighTemperature Application. Entropy 2019, 21, 1058. [CrossRef]

3. Feldhoff, A. Thermoelectric Material Tensor Derived from the Onsager-de Groot-Callen Model. Energy Harvest. Syst. 2015, 2, 5-13. [CrossRef]

4. Feldhoff, A. Power Conversion and Its Efficiency in Thermoelectric Materials. Entropy 2020, 22, 803. [CrossRef]

5. Fuchs, H.U. The Dynamics of Heat-A Unified Approach to Thermodynamics and Heat Transfer, 2nd ed.; Springer: New York, NY, USA, 2010.

6. Ioffe, A.F. Semiconductor Thermoelements, and Thermoelectric Cooling, 1st ed.; Info-Search Ltd.: London, UK, 1957.

7. Tan, G.; Zhao, L.D.; Kanatzidis, M.G. Rationally Designing High-Performance Bulk Thermoelectric Materials. Chem. Rev. 2016, 116, 12123-12149. [CrossRef]

8. Shi, X.; Chen, L.; Uher, C. Recent advances in high-performance bulk thermoelectric materials. Int. Mater. Rev. 2016, 61, 379-415. [CrossRef]

9. Kanatzidis, M.G. Nanostructured thermoelectrics: The new paradigm? Chem. Mater. 2010, 22, 648-659. [CrossRef]

10. Poudel, B.; Hao, Q.; Ma, Y.; Lan, Y.; Minnich, A.; Yu, B.; Yan, X.; Wang, D.; Muto, A.; Vashaee, D.; et al. High-Thermoelectric Performance of Nanostructured Bismuth Antimony Telluride Bulk Alloys. Science 2008, 320, 634-638. [CrossRef]

11. Ashalley, E.; Chen, H.; Tong, X.; Li, H.; Wang, Z.M. Bismuth telluride nanostructures: Preparation, thermoelectric properties and topological insulating effect. Front. Mater. Sci. 2015, 9, 103-125. [CrossRef]

12. Bittner, M.; Helmich, L.; Nietschke, F.; Geppert, B.; Oeckler, O.; Feldhoff, A. Porous $\mathrm{Ca}_{3} \mathrm{Co}_{4} \mathrm{O}_{9}$ with enhanced thermoelectric properties derived from Sol-Gel synthesis. J. Eur. Ceram. Soc. 2017, 37, 3909-3915. [CrossRef]

13. Delorme, F.; Giovannelli, F. Effect of Ca substitution by Fe on the thermoelectric properties of $\mathrm{Ca}_{3} \mathrm{Co}_{4} \mathrm{O}_{9}$ ceramics. J. Electroanal. Chem. Interfacial Electrochem. 2018, 40, 107-114. [CrossRef] 
14. He, J.; Liu, Y.; Funahashi, R. Oxide thermoelectrics: The challenges, progress, and outlook. J. Mater. Res. 2011, 26, 1762-1772. [CrossRef]

15. Imasato, K.; Kang, S.D.; Snyder, G.J. Exceptional thermoelectric performance in $\mathrm{Mg}_{3} \mathrm{Sb}_{0.6} \mathrm{Bi}_{1.4}$ for low-grade waste heat recovery. Energy Environ. Sci. 2019, 12, 965-971. [CrossRef]

16. Toberer, E.S.; May, A.F.; Snyder, G.J. Zintl chemistry for designing high efficiency thermoelectric materials. Chem. Mater. 2010, 22, 624-634. [CrossRef]

17. Poon, J.S. Recent Advances in Thermoelectric Performance of Half-Heusler Compounds. Metals 2018, 8, 989. [CrossRef]

18. Chen, S.; Ren, Z. Recent progress of half-Heusler for moderate temperature thermoelectric applications. Mater. Today 2013, 16, 387-395. [CrossRef]

19. Zhu, H.; He, R.; Mao, J.; Zhu, Q.; Li, C.; Sun, J.; Ren, W.; Wang, Y.; Liu, Z.; Tang, Z.; et al. Discovery of ZrCoBi based half Heuslers with high thermoelectric conversion efficiency. Nat. Commun. 2018, 9, 2497. [CrossRef] [PubMed]

20. Narducci, D. Do we really need high thermoelectric figures of merit? A critical appraisal to the power conversion efficiency of thermoelectric materials. Appl. Phys. Lett. 2011, 99, 102104. [CrossRef]

21. Bittner, M.; Kanas, N.; Hinterding, R.; Steinbach, F.; Groeneveld, D.; Wemhoff, P.; Wiik, K.; Einarsrud, M.A.; Feldhoff, A. Triplephase ceramic 2D nanocomposite with enhanced thermoelectric properties. J. Eur. Ceram. Soc. 2019, 39, 1237-1244. [CrossRef]

22. Xu, Z.J.; Hu, L.P.; Ying, P.J.; Zhao, X.B.; Zhu, T.J. Enhanced thermoelectric and mechanical properties of zone melted $p$-type $(\mathrm{Bi}, \mathrm{Sb})_{2} \mathrm{Te}_{3}$ thermoelectric materials by hot deformation. Acta Mater. 2015, 84, 385-392. [CrossRef]

23. Fu, C.; Bai, S.; Liu, Y.; Tang, Y.; Chen, L.; Zhao, X.; Zhu, T. Realizing high figure of merit in heavy-band $p$-type half-Heusler thermoelectric materials. Nat. Commun. 2015, 6, 8144. [CrossRef]

24. Muta, H.; Kurosaki, K.; Uno, M.; Yamanaka, S. Thermoelectric Properties of constantan/spherical $\mathrm{SiO}_{2}$ and $\mathrm{Al}_{2} \mathrm{O}_{3}$ particles composite. J. Alloys Compd. 2003, 359, 326-329. [CrossRef]

25. Mao, J.; Wang, Y.; Kim, H.S.; Liu, Z.; Saparamadu, U.; Tian, F.; Dahal, K.; Sun, J.; Chen, S.; Liu, W.; et al. High thermoelectric power factor in Cu-Ni alloy originate from potential barrier scattering of twin boundaries. Nano Energy 2015, 17, 279-289. [CrossRef]

26. Wiendlocha, B. Thermopower of thermoelectric materials with resonant levels: PbTe:Tl versus PbTe:Na and Cu1-xNix. Phys. Rev. B 2018, 97, 205203. [CrossRef]

27. Wolf, M.; Menekse, K.; Mundstock, A.; Hinterding, R.; Nietschke, F.; Oeckler, O.; Feldhoff, A. Low Thermal Conductivity in Thermoelectric Oxide-Based Multiphase Composites. J. Electron. Mater. 2019, 48, 7551-7561. [CrossRef]

28. Liu, W.; Kim, H.S.; Chen, S.; Jie, Q.; Lv, B.; Yao, M.; Ren, Z.; Opeil, C.P.; Wilson, S.; Chu, C.W.; et al. n-type thermoelectric material $\mathrm{Mg}_{2} \mathrm{Sn}_{0.75} \mathrm{Ge}_{0.25}$ for high power generation. Proc. Natl. Acad. Sci. USA 2015, 112, 3269-3274. [CrossRef]

29. Yuan, M.; Sun, L.; Lu, X.W.; Jiang, P.; Bao, X.H. Enhancing the thermoelectric performance of Cu-Ni alloys by introducing carbon nanotubes. Mater. Today Phys. 2021, 16, 100311. [CrossRef]

30. Kang, H.; Yang, Z.; Yang, X.; Li, J.; He, W.; Chen, Z.; Guo, E.; Zhao, L.D.; Wang, T. Preparing bulk Cu-Ni-Mn based thermoelectric alloys and synergistically improving their thermoelectric and mechanical properties using nanotwins and nanoprecipitates. Mater. Today Phys. 2021, 17, 100332. [CrossRef]

31. Shimizu, Y.; Mizoshiri, M.; Mikami, M.; Sakurai, J.; Hata, S. Fabrication of Copper/Copper-Nickel thin-film thermoelectric generators with energy storage devices. J. Phys. Conf. Ser. 2018, 1052, 012032. [CrossRef]

32. Lide, D.R. CRD Handbook of Chemistry and Physics, 89th ed.; CRC Press/Taylor and Francis: Boca Raton, FL, USA, 2009.

33. Haubold, T.; Gertsman, V. On the structure and properties of nanostructured copper-tungsten alloys. Nanostruct. Mater. 1992, 1, 303-312. [CrossRef]

34. Steinhoff, T.; Wolf, M.; Nürnberger, F.; Gerstein, G.; Feldhoff, A. Evaluation of Cu-Ni-based alloys for thermoelectric energy conversion. Mater. Sci. Forum 2021, 1016, 107-112. [CrossRef]

35. Wang, J.; Wei, L.; Zhang, L.; Zhang, J.; Wei, H.; Jiang, C.; Zhang, Y. Zinc-doped nickel oxide dendritic crystals with fast response and self-recovery for ammonia detection at room temperature. J. Mater. Chem. 2012, 22, 20038-20047. [CrossRef]

36. Zhuo, K.; Jeong, M.G.; Chung, C.H. Dendritic nanoporous nickel oxides for a supercapacitor prepared by a galvanic displacement reaction with chlorine ions as an accelerator. RSC Adv. 2013, 3, 12611-12615. [CrossRef]

37. Wang, F.; Lu, Y.; Zeng, S.; Song, Y.; Zheng, D.; Xu, W.; Lu, X. Nickel@Nickel Oxide Dendritic Architectures with Boosted Electrochemical Reactivity for Aqueous Nickel-Zinc Batteries. ChemElectroChem 2020, 7, 4572-4577. [CrossRef] 